\title{
Maternal and Fetal Outcome in Antepartum Haemorrhage of Unknown Origin in Chennai, India
}

\author{
Swetha Gulabi Gaddam ${ }^{1}$, Vijithra Thangamani² \\ 1, 2 Department of Obstetrics and Gynaecology, Sri Ramachandra Institute of Higher \\ Education and Research, Porur, Chennai, Tamil Nadu, India.
}

\section{ABSTRACT}

\section{BACKGROUND}

Antepartum haemorrhage of unknown origin (APHUO) being a diagnosis of exclusion, is a rare condition which poses dilemma in the management of pregnancy in terms of timing and mode of delivery. The purpose of this study was to evaluate antenatal factors associated with APHUO, clinical presentation and analyse its impact on pregnancy and its outcome.

\section{METHODS}

This is a retrospective study conducted over a period of two years in a tertiary care hospital. Pregnancy outcomes were compared between 41 cases who had APHUO versus 39 controls who never had history of bleeding in their antepartum period. Bleeding pattern, incidence of preterm labour, intra partum and postpartum complications, mode of delivery, birth weight, APGAR (appearance, pulse, grimace, activity, and respiration) score of the baby and neo-natal intensive care unit (NICU) admission were analysed.

\section{RESULTS}

Patients with APHUO had subclinical abruption and increased risk of preterm delivery. Intrapartum and postpartum complications were similar among both the groups. The average birth weight was much lesser in the study group, but the cause was attributed to prematurity. These findings were consistent with the previous studies.

\section{CONCLUSIONS}

APHUO is associated with subclinical abruption and increased risk of preterm labour. Hence the patient should be counselled for delivery at a tertiary care center with adequate neonatal care. Greater incidence of NICU admission and low birth weight were attributed to prematurity among the study group. Induction of labour at term in this group is of questionable value unless there is an associated obstetric indication.

\section{KEY WORDS}

APHUO, Preterm Labour, Sub Clinical Abruption, Low Birth Weight
Corresponding Author: Dr. Vijithra Thangamani, Department of Obstetrics and Gynaecology, Sri Ramachandra Institute of Higher Education and Research, Porur, Chennai, Tamil Nadu, India. E-mail:vijithra.t@gmail.com

DOI: $10.14260 / \mathrm{jemds} / 2021 / 507$

How to Cite This Article: Gaddam SG, Thangamani V. Maternal and fetal outcome in antepartum haemorrhage of unknown origin in Chennai, India. J Evolution Med Dent Sci 2021;10(31):24812484, DOI: $10.14260 /$ jemds/2021/507

Submission 17-02-2021,

Peer Review 20-05-2021,

Acceptance 26-05-2021,

Published 02-08-2021.

Copyright (c) 2021 Swetha Gulabi Gaddam et al. This is an open access article distributed under Creative Commons Attribution License [Attribution 4.0 International (CC BY 4.0)] 


\section{BACKGROUND}

Antepartum haemorrhage is defined as bleeding from or into the genital tract occurring from the period of viability of pregnancy till the delivery of the baby, ${ }^{1}$ wherein period of viability varies among different countries [according to world health organization (WHO) it is after 22 weeks or fetus weight more than 500 grams]. The incidence ranges from $0.5-5 \%$ of pregnancies which depends on socio demographic variables..$^{2,3}$ Maternal complications of antepartum haemorrhage include preterm labour, anaemia, increased incidence of caesarean section, increased incidence of thromboembolic manifestations, postpartum haemorrhage $(\mathrm{PPH})$, peripartum hysterectomy, coagulation failure, puerperal infections etc. It can even lead to maternal mortality. The two most important causes are placenta previa and abruptio placentae (although these are not the most common causes), ${ }^{3}$ the other causes include local lesions of the cervix like cervicitis, cervical erosion, ectropion, cervical polyp, cervical cancer, vaginal lesions and vulvar varicosities, abdominal trauma, genital trauma, prelabour rupture of membrane, ruptured vasa previa.1,4,5 However, in few cases the cause cannot be determined even after performing a thorough clinical and ultrasound examination, hence labelled as antepartum haemorrhage of unknown origin (APHUO). ${ }^{3}$ Heavy show is also included in APH. ${ }^{3}$ The common causes of APH can be ruled out by thorough history and clinical examination which includes general examination, abdominal and vaginal speculum examination. However, the diagnosis is confirmed by ultrasound. If clinical suspicion arises, blood investigations are done to rule out coagulopathies. In any case of antepartum haemorrhage there must be a stepwise approach.

Abruptio placenta and local lesions can be diagnosed clinically whereas placenta previa needs confirmation by ultrasound (either transabdominal or transvaginal, the latter being more sensitive in diagnosing placenta previa, can even be done during active bleeding). Apart from causing maternal morbidity and mortality, antepartum haemorrhage is associated with significant neonatal morbidity and mortality because of preterm delivery and acute hypoxia. Of all the very preterm babies, one fifth are born in association with antepartum haemorrhage. Antepartum haemorrhage is also associated with increased incidence of cerebral palsy; however, the cause is attributed to prematurity.

Though antepartum haemorrhage of unknown origin is associated with minimum bleeding and in most of the cases it is non recurrent and does not need active intervention, and hence it is worrisome for the treating obstetrician and the patient. Hence various studies have been conducted regarding APHUO and its complications. The purpose of this study was to evaluate clinical presentation and antenatal factors associated with APHUO and analyse its impact on the pregnancy and its outcome.

\section{METHODS}

A retrospective data analysis of all patients diagnosed as having antepartum haemorrhage of unknown origin over a period of two years from January 2017 to December 2018 was performed. The known causes of APH were ruled out by thorough history and clinical examination, vaginal speculum examination, ultrasound examination and blood investigations to rule out coagulopathies. A case-control study was performed, with controls being chosen from the computer database.

The controls were matched for age and parity which could be the major individual risk factors affecting the incidence of antepartum haemorrhage. Singleton pregnancies who never had a history of bleeding in the antenatal period and delivered during the same period of time as the study cases were included in the study. Analysis was performed between the two groups and the following variables were included in the analysis - gestational age at presentation, bleeding pattern (quantity of blood loss and recurrence), incidence of preterm labour, intra partum and postpartum complications, mode of delivery, associated subclinical abruption, birth weight and APGAR score of the baby and NICU admission.

\section{Statistical Analysis}

Independent $\mathrm{t}$ - tests were used for continuous variables after verifying equality of variances and discrete parameters were compared using two tailed chi square and Fisher exact tests. A $\mathrm{P}$ - value of less than 0.5 was considered statistically significant.

\section{RESULTS}

Among 41 patients included in the study, $43.9 \%$ were primi gravida, $56.1 \%$ were multigravida (Table 1 ). Since it was a single center study, socio demographic characteristics were the same for both groups. Mean age of patients presenting with APHUO was 29.1 years and that for the control group was 28.9 years. The mean gestational age at which patients presented with APHUO was at 31 weeks. Smoking incidence in our study population did not differ between two groups.

\begin{tabular}{|ccc|}
\hline Parity & Cases & Controls \\
Primi & $18(43.9 \%)$ & $17(43.6 \%)$ \\
Multi & $23(56.1 \%)$ & $22(56.4 \%)$ \\
Total & $\mathbf{4 1}$ & $\mathbf{3 9}$ \\
\hline Table 1. Distribution of Study Population Based on Parity \\
\hline
\end{tabular}

In our study, $80 \%$ of the patients presented with only spotting per vaginum while the remaining $20 \%$ had soakage of a pad. None of the patients presented with heavy bleeding which needed an immediate active intervention. On analysing the pattern of recurrence, among the case group, $80 \%$ of the patients had only one episode of spotting or bleeding per vaginum while the remaining $20 \%$ had recurrent episodes. However, none of them had heavy bleeding. Of the patients who presented with APHUO, 7.3 \% had anaemia but the cause wasn't attributed to bleeding, it was due to other common causes like nutritional anaemia. Among the control group, 2.6 $\%$ had anaemia (Table 2).

There were no intra partum complications among both the groups. However, $7.3 \%$ had postpartum haemorrhage among the cases while no one in the control group had postpartum complications. But it was not statistically significant. Among the cases, $14.6 \%$ had subclinical abruption which was statistically significant whereas it was nil in control group 
(Table2.1). Regarding the mode of delivery among the cases, $36.6 \%$ delivered vaginally; $63.4 \%$ underwent lower segment caesarean section (LSCS). Among the control group, $41 \%$ delivered vaginally; $59 \%$ underwent LSCS (Table 3). The number of inductions of labour and indications for LSCS are discussed below.

\begin{tabular}{|ccc|}
\hline Anaemia & Cases & Control \\
\hline Present & $3(7.3 \%)$ & $1(2.6 \%)$ \\
Absent & $38(92.7 \%)$ & $38(97.4 \%)$ \\
\hline Table 2. Distribution of Patients with Anaemia in the Study Population
\end{tabular}

\begin{tabular}{|ccc|}
\hline Subclinical Abruption & Cases & Control \\
Present & $6(14.6 \%)$ & 0 \\
Absent & $35(85.4 \%)$ & $39(100 \%)$ \\
(P value $=0.013)$ & \\
\hline \multicolumn{2}{|c|}{ Table 2.1. Distribution of Patients with Subclinical Abruption } \\
in the Study Population
\end{tabular}

\begin{tabular}{|ccc|}
\hline Mode of Delivery & Cases & Control \\
SVD & $15(36.6 \%)$ & $16(41 \%)$ \\
LSCS & $26(63.4 \%)$ & $23(59 \%)$ \\
Total & $\mathbf{4 1}$ & $\mathbf{3 9}$ \\
\hline \multicolumn{2}{|c|}{ Table 3. Distribution of Mode of Delivery among } \\
& the Case and Control Groups \\
\hline
\end{tabular}

The incidence of preterm labour in the case group $(n=41)$ was $41.4 \%$ while that in the control group $(n=39)$ was 20.5 $\%$. Among the case group, mean gestation age at delivery was 35 weeks. In the control group, mean gestation age at delivery was at 37 weeks. In the case group, among the patients who underwent LSCS, 13 patients had a preterm delivery. Among these 13 patients, the indication for delivery was APHUO, whereas three patients had fetal distress on cardiotocography (CTG) monitoring. The other indications were previous LSCS in labour, pre-term premature rupture of the membranes (PPROM) and eclampsia.

In the control group who underwent LSCS, seven patients were delivered as preterm with indications being one of the following - previous LSCS in labour, PPROM, intra uterine growth restriction (IUGR) with Doppler changes (Table 4).

\begin{tabular}{|ccc|}
\hline Indication for LSCS & Cases & Control \\
Failed induction & $3(11.5 \%)$ & $9(39 \%)$ \\
Fetal distress & $3(11.5 \%)$ & $4(17.3 \%)$ \\
Previous LSCS & $8(30.7 \%)$ & $6(26 \%)$ \\
\hline PPROM & $2(7.7 \%)$ & $2(8.6 \%)$ \\
IUGR & 0 & $2(8.6 \%)$ \\
APHUO & $10(38.4 \%)$ & 0 \\
Total & $\mathbf{2 6}$ & $\mathbf{2 3}$ \\
\hline Table 4. Indication for LSCS among the Cases and Control Group \\
\hline
\end{tabular}

The number of inductions of labour did not differ in both groups. In the case group it was $27.5 \%$, the most common indication being term pregnancy followed by prelabour rupture of membranes (PROM); while in the control group it was $36 \%$. The most common indication being the same as that of case group (Table 5). The other indications were gestational diabetes mellitus (GDM), decreased fetal movements, oligohydramnios, labour abnormalities like prolonged latent phase, etc.

The overall birth weight was low in the case group, the mean being $2.54 \mathrm{~kg}$, while for the control group the mean weight was $2.88 \mathrm{~kg}$. This disparity is due to iatrogenic preterm delivery among the patients with APHUO. The APGAR scores at one minute and five minutes were compared among the cases and controls. There was not much difference in the numbers among both the groups. The number of admissions to neonatal intensive care units were more in those with APHUO (39\%) while it was only $17.9 \%$ among the control group. Though statistically significant, increased risk of admission to NICU was due to complications associated with preterm delivery (Table 5.1).

\begin{tabular}{|ccc|}
\hline Indication for IOL & Cases & Control \\
Term & $4(36.3 \%)$ & $5(35.7 \%)$ \\
PROM & $3(27.2 \%)$ & $1(7.1 \%)$ \\
GDM & 0 & $3(21.4 \%)$ \\
Decreased fetal movements & $2(18.1 \%)$ & $1(7.1 \%)$ \\
Oligohydramnios & $2(18.1 \%)$ & $1(7.1 \%)$ \\
Prolonged latent phase & 0 & $3(21.4 \%)$ \\
Total & $\mathbf{1 1}$ & $\mathbf{1 4}$ \\
\hline Table 5. Indications for Induction of Labour \\
among the Case and Control Groups \\
\hline
\end{tabular}

\begin{tabular}{|cc|c|}
\hline NICU Admission & Cases & Control \\
Yes & $16(39 \%)$ & $7(17.9 \%)$ \\
No & $25(61 \%)$ & $32(82.1 \%)$ \\
\hline \multicolumn{2}{|c|}{ Table 5.1. Distribution of NICU Admissions } \\
\hline \multicolumn{2}{|c}{} \\
\hline
\end{tabular}

Antepartum haemorrhage of unknown origin does not pose any significant risk to the fetus in intrauterine period. There was no stillbirth among both the groups.

\section{DISCUSSION}

The incidence of APHUO was reported to be around $2-10$ $\% .6,7,8,9$ However the incidence varies widely based on the socio-demographic variables. The known causes of antepartum haemorrhage are associated with conditions occurring during pregnancy such as placenta previa, abruptio placenta, and local genital lesions, abdominal trauma, intrauterine infections, pre labour rupture of membranes, polyhydramnios, elevated maternal serum alpha - fetoprotein, smoking, and substance abuse, etc. ${ }^{3}$ Similarly some of the risk factors for APHUO include vaginal bleeding in early pregnancy, overweight, obesity, smoking. ${ }^{7}$

Few studies have shown APHUO is associated with smoking, however study done by Marlies et al. showed when adjusted for confounders, smoking was no longer significantly associated with APHUO.7 In our study smoking incidence was same among cases and controls, hence its association with APHUO could not be studied. Patients who presented with first trimester bleeding were excluded from the study. There was no significant difference in the body mass index (BMI) of both the study groups. Hence no specific risk factor was identified with APHUO as per this study.

Maternal complications of antepartum haemorrhage include hypovolemic shock, higher rates of caesarean section, peripartum hysterectomies, disseminated intravascular coagulation (DIC), multiple blood and blood products transfusion, ICU care, post-operative anaemia etc. The maternal mortality rate being $1 \%{ }^{10,11,12}$ There are no major maternal complications associated with APHUO except for increased frequency of labour inductions at term, iatrogenic preterm delivery, and emergency LSCS which is consistent with previous studies.

Fetal complications of APH include premature delivery, low birth weight, birth asphyxia, and intrauterine fetal death, 
hyperbilirubinemia. ${ }^{1}$ Fetal complications associated with APHUO are preterm delivery, which in most cases was iatrogenic, NICU admission and small for birth weight babies (Willock et al.). ${ }^{13}$ It was proven in the current study, however birth weight was same as that of controls when adjusted for gestational age. A study done by Magann et al. showed increased incidence of stillbirth and fetal anomalies in patients with APHUO, 6 there was no such finding in the current study.

Yeung et al. conducted a study to determine the rate and risk factors, predicting preterm delivery among the women presenting with APHUO before 34 weeks. They concluded that only those who had two or more episodes of APHUO or persistent bleeding had increased incidence of preterm labour and also that APHUO prior to 34 weeks of gestation is associated with three to five-fold increased risk of preterm labour. ${ }^{14}$ In our study, $80 \%$ of the patients had only one episode of APHUO and the other $20 \%$ had recurrent episodes, but incidence of preterm labour was similar in both the groups.

Claudine Hartell conducted a study at TygerBerg Hospital, South Africa. The incidence of APHUO in their study was $2.6 \%$. They have observed that in $8.1 \%$ of patients presenting with APHUO, Abruptio placentae was diagnosed on placental examination. ${ }^{8}$ This was proven in our study wherein $14.6 \%$ had subclinical abruption which warrants thorough examination of placenta and also to anticipate retro placental clot in patients presenting with APHUO immediately after delivery. A retrospective study done by Ogueh et al. showed that there is no need for hospitalizing patients presenting with APHUO without any other associated obstetric complications and in the absence of heavy or repeated bleeding, evidence of fetal or maternal compromise or onset of labour. ${ }^{15}$ The incidence of preterm labour in patients presenting with APHUO is much higher than those who never had a history of bleeding in the antenatal period ( $41.4 \%$ vs $20.5 \%$ ). This finding is compatible with the previous studies. ${ }^{8,9,16}$

\section{CONCLUSIONS}

APHUO is associated with an increased risk of preterm labour. Hence, the patient should be counselled for delivery at a tertiary care center with adequate neonatal care. Subclinical abruption is found in a significant proportion of patients with APHUO. Greater incidence of NICU admission and low birth weight were attributed to prematurity among the study group. Induction of labour at term in this group is of questionable value unless there is an associated obstetric indication.

Data sharing statement provided by the authors is available with the full text of this article at jemds.com.

Financial or other competing interests: None.

Disclosure forms provided by the authors are available with the full text of this article at jemds.com.

\section{REFERENCES}

[1] Arulkumaran S, Regan L. Antepartum haemorrhage. In: Obstetrics and Gynaecology. 1 ${ }^{\text {st }}$ edn. New York: USA: Oxford University Press 2011:326-7.

[2] Singhal S, Nymphaea, Nanda S. Maternal and perinatal outcome in antepartum haemorrhage: a study at a tertiary care referral institute. Internet J Gynaecol Obstet 2007;9(2):1-4.

[3] Antepartum Haemorrhage (Green-top Guideline No. 63). London: Royal College of Obstetricians and Gynaecologists 2011.

[4] Takai IU, Sayyadi BM, Galadanci HS. Antepartum hemorrhage: a retrospective analysis from a Northern Nigerian teaching hospital. Int J Appl Basic Med Res 2017;7(2):112-6.

[5] Van Calsteren K, Vergote I, Amant F. Cervical neoplasia during pregnancy: diagnosis, management and prognosis. Best Pract Res Clin Obstet Gynaecol 2005;19(4):611-30.

[6] Magann EF, Cummings JE, Niederhauser A, et al. Antepartum bleeding of unknown origin in the second half of pregnancy: a review. Obstet Gynecol Surv 2005;60(11):741-5.

[7] Van Altvorst MEWA, Chan EHY, Taylor RS, et al. Antepartum haemorrhage of unknown origin and maternal cigarette smoking beyond the first trimester. Aust New Z J Obstet Gynaecol 2012;52(2):161-6.

[8] Hartell C. The incidence and outcomes of antepartum haemorrhage of unknown origin at Tygerberg hospital: a retrospective, descriptive study. Stellenbosch University 2017.

[9] Bhandari S, Raja EA, Shetty A, et al. Maternal and perinatal consequences of antepartum haemorrhage of unknown origin. BJOG 2014;121(1):44-50.

[10] Pandelis K, Kardina A, Samina M. Antepartum haemorrhage. Obstet Gynaecol Reprod Med 2012;1:21-5.

[11] Kavita C, Chavda R. Maternal and perinatal outcome in antepartum haemorrhage. Int $\mathrm{J}$ Obstet Gynaecol 2019;3(1):221-5.

[12] Borodo AT, Shehu CE. Placenta previae at Usmanu Danfodio University teaching hospital: a 5 year review. Sahel Med J 2013;16(2):56-9.

[13] Willocks J. Antepartum haemorrhage of uncertain origin. J Obstet Gynaecol Br Commonw 1971;78(11):987-91.

[14] Yeung SW, Tam WH, Cheung RY. The risk of preterm delivery prior to 34 weeks in women presenting with antepartum haemorrhage of unknown origin. Aust N Z J Obstet Gynaecol 2012;52(2):167-72.

[15] Ogueh 0 , Johnson MR. What is the value of hospitalisation in antepartum haemorrhage of uncertain origin? J Obstet Gynaecol 1998;18(2):120-2.

[16] Chan CC, To WW. Antepartum hemorrhage of unknown origin--what is its clinical significance? Acta Obstet Gynecol Scand 1999;78(3):186-90. 